\title{
Innovación de la Práctica Docente en la UNAE, Ecuador recurriendo a la Web 2.0
}

Autores: Gladys Isabel Portilla Faicán Universidad Nacional de Educación, UNAE gladys.portilla@unae.edu.ec Azogues, Ecuador Amauris Laurencio Leyva Universidad de La Habana, UH amalaur@cepes.uh.cu La Habana, Cuba

Resumen

En este trabajo se socializa una experiencia de innovación de la práctica docente desarrollada en la Universidad Nacional de Educación (UNAE) en la asignatura Sociedad y Cultura. Se identificó que los estudiantes tenían dificultad para comprender problemática disciplinares como el tiempo histórico, la explicación multicausal, los actores colectivos, entre otros temas. En la lógica de la investigación-acción, en el colectivo de la asignatura se formó una comunidad de aprendizaje como estrategia de gestión de la innovación de la práctica docente. Para abordar el problema se recurrió a la metodología Lesson Study. Los procesos de enseñanza-aprendizaje fueron orientados por el principio pedagógico-metodológico del Flipped classroom y los enfoques pedagógicos del Modelo Pedagógico de la UNAE, el constructivismo, enactivismo y conectivismo. El objetivo fue innovar la práctica docente para desarrollar la comprensión de temas curriculares fundamentales de la Historia aprovechando el potencial educativo de la Web 2.0.

Palabras clave: innovación pedagógica; investigación pedagógica; tecnología educacional. 


\title{
Innovation of the Teaching Practice in the UNAE, Ecuador recurring to Web 2.0
}

\begin{abstract}
In this paper is socialized an innovative approach of teaching practice developed at the Universidad Nacional de Educación (UNAE) in the Society and Culture subject. It was identified that students had difficulty to understand problematic disciplines such as historical time, multi-causal explanation, collective actors, among other topics. In the logic of action research, in the collective subject Teachers was created a learning community as a management strategy for the innovation of teaching practice. To address the problem, the Lesson Study methodology was used. The teaching-learning processes were guided by the pedagogical-methodological principle of the overturned classroom and the pedagogical approaches of the Pedagogical Model of UNAE, such as constructivism, enactivism and connectivism. The objective was to innovate the teaching practice to develop the comprehension of fundamental curricular topics of History taking advantage of the educational potential of the Web 2.0.
\end{abstract}

Keywords: pedagogical innovation; pedagogical research; educational technology. 


\section{Introducción}

En este trabajo se presenta un resultado de una concepción teóricometodológica para innovar la práctica docente recurriendo al potencial innovador de la Web 2.0, en el contexto de la UNAE. Una de las competencias profesionales del docente contemporáneo planteadas en el Modelo Pedagógico de la UNAE es la capacidad para diseñar y construir contextos y comunidades de aprendizaje (Sanz y Pérez, 2009; Comisión Gestora de la Universidad Nacional de Educación, 2015a).

La asignatura Sociedad y Cultura, parte del currículo de nivelación de carreras de educación en Ecuador, es una síntesis de las ciencias sociales en el currículo del bachillerato ecuatoriano, que tiene como como eje pedagógico el desarrollo del pensamiento histórico para la comprensión de los sucesos a lo largo de la Historia. La reforma curricular del bachillerato implementado en el 2012 por el (Ministerio de Educación de Ecuador, 2012), trajo consigo el desafío de innovar los procesos de enseñanza-aprendizaje $y$, en consecuencia, la práctica docente. A pesar de los esfuerzos para implementar el currículo los estudiantes aún llegan a la Universidad con muchas dificultades para afrontar el desafío cognitivo de comprender el tiempo histórico.

En el colectivo de la asignatura se identificó como una problemática fundamental para el desarrollo del pensamiento histórico de los jóvenes, la capacidad para comprender la relevancia de la narrativa histórica, a partir de identificar las continuidades y discontinuidades del tiempo histórico, los actores colectivos, la multicausalidad, entre otras habilidades disciplinares. Como resultado de la evaluación diagnóstica de los saberes previos de los estudiantes se identificó la carencia de estas habilidades, de cara al imperativo de "fomentar el desarrollo de una mirada histórica del presente, a través del análisis informado de la historia de la humanidad, con especial énfasis en la de nuestro país y la región" (Sistema Nacional de Nivelación y Admisión, 2014, pág. 16). 
En este contexto, en el colectivo de la asignatura se formó una comunidad de aprendizaje, con el propósito de innovar los procesos de enseñanza-aprendizaje. El objetivo de la investigación de la propia práctica docente fue desarrollar en los estudiantes la comprensión de las nociones de temporalidad, multicausalidad y actoría colectiva en los sucesos históricos, para entender el presente histórico recurriendo al uso innovador de tecnologías de la WEB 2.0.

El colectivo constituido como comunidad de aprendizaje, decidió mejorar la práctica docente a través de la metodología Lesson Study. Esta metodología contempla sietes fases: 1). Definir el problema, 2). Diseñar cooperativamente una "lección experimental", 3). Enseñar y observar la lección, 4). Recoger las evidencias y discutir, 5). Analizar y revisar la lección, 6). Desarrollar la lección revisada en otra clase y observar de nuevo, 7). Discutir, evaluar y reflexionar sobre las nuevas evidencias y diseminar la experiencia (Soto y Pérez, 2014a).

En la comunidad de práctica docente, con base en la problemática, encontrada se diseñaron tres lecciones, en las que metodológicamente se observó, analizo y planificó las lecciones mejoradas y sucesivas. Se recurrió a la Lesson Study definida como una metodología de investigación y mejora de la propia práctica educativa (Soto, 2014b), como un proceso permanente de la reflexión sobre la acción (Comisión Gestora de la Universidad Nacional de Educación, 2015b). El diseño de las lecciones se centró en los procesos de enseñanza-aprendizaje de las temáticas disciplinares que implican habilidades de pensamiento histórico.

El diseño, rediseño e implementación de tres lecciones resultaron muy eficaz y altamente innovador para el aprendizaje de la Historia en el contexto de la era digital. El proceso de diseñar y desarrollar en equipo la Lesson Study implicó un excelente espacio para la reflexión colectiva de la propia práctica docente. Fue muy enriquecedor asumir de forma alternada los roles de 
diseñadores, docente conductor de la lección, observador y luego todos de revisores. El análisis de la práctica generó espacios de socialización del colectivo sobre lo observado (por los registradores) y lo implementado por el docente ejecutor de la práctica. Los ambientes y escenarios de aprendizaje, las interrelaciones personales, los roles, los lenguajes y recursos para la presentación y comunicación de los logros de aprendizaje dieron cuenta del impacto positivo de la innovación desarrollada a través de la metodología Lesson Study.

\section{Metodología}

A partir de la identificación de la problemática de aprendizaje de los estudiantes, la primera acción del colectivo fue la conformación de una comunidad de aprendizaje. Para afrontar el desafío de innovar la práctica docente y mejorar los aprendizajes se procedió a investigar cooperativamente la propia práctica docente recurriendo a la Lesson Study como metodología de investigación para la innovación y la mejora. Se diseñó una Lesson Study basada en la información de la evaluación diagnóstica de las experiencias previas de aprendizaje de los estudiantes y las habilidades de pensamiento. En la Lesson Study participaron 158 estudiantes y cuatro docentes del colectivo. Se diseñó, rediseñó y desarrolló las lecciones de forma cooperativa e involucrando tecnología educativa de la Web 2.0, concebida como un elemento motivante, dinamizador y generador de aprendizajes colectivos, situados y auténticos.

El método de la secuencia didáctica con la que se desarrolló el diseño de las lecciones fue el aprendizaje basado en proyectos (ABP). En la fase de experimentación de cada una de las tres lecciones se hizo observación directa y a través de filmaciones del desarrollo de las lecciones. En la fase previa y posterior al desarrollo de la Lesson Study se aplicó encuestas de evaluación diagnóstica y de resultados, así como también la técnica de grupo focal, para 
evaluar la situación inicial y la posterior, como resultados del impacto de la metodología en los aprendizajes alcanzados por los estudiantes.

\section{Resultados y discusión}

La UNAE emerge en el sistema educativo ecuatoriano como una estrategia para fomentar la excelencia educativa (Asamblea Constituyente, 2008; Ministerio de Educación del Ecuador, 2012). En concordancia con este marco normativo el Modelo Pedagógico de la UNAE tiene como uno de sus principios pedagógicos "Promover la didáctica invertida, el Flipped classroom, las redes sociales virtuales y las plataformas digitales" (Comisión Gestora de la Universidad Nacional de Educación, 2015c), recurriendo a una estrategia metodológica innovadora, como la Lesson Study (Soto y Pérez, 2014c).

La problemática de lograr la comprensión del presente como algo histórico es uno de los principios didáctico-disciplinares de la Historia (Vallejo, 2008; Ministerio de Educación del Ecuador, 2010). La comprensión de las nociones de temporalidad en el análisis de los eventos históricos implica identificar continuidades y discontinuidades, que explican el presente y el legado histórico como un elemento fundamental de los mismos.

Con el fin de tener una lectura de los conocimientos previos y habilidades de pensamiento de los estudiantes, en torno a la Historia, se aplicó una encuesta a todos ellos al inicio del semestre. Los resultados del análisis de la encuesta y de las primeras evaluaciones de los procesos de aprendizaje en la asignatura evidenciaron que los estudiantes presentaban dificultades para comprender el presente como histórico, lo que implica la comprensión del tiempo histórico, los actores y procesos que explican los sucesos históricos, pues estaban habituados a hacer una lectura lineal de la Historia, en la que los eventos se suceden y pasan a ser parte de la Historia, con figuras icónicas y explicaciones superficiales. Esto se evidenció en la evaluación diagnóstica. Ante el desafío de desarrollar el pensamiento histórico de los estudiantes 
surgió la propuesta de diseñar un proceso de enseñanza aprendizaje orientado a desarrollar la comprensión de la Historia, en su complejidad.

\subsection{Lección experimental}

Según el proceso metodológico de la Lesson Study, propuesto por Soto y Pérez (2014d), se definió el problema en el colectivo y se diseñó una lección experimental (una secuencia didáctica), que tuvo como objetivo de aprendizaje, caracterizar las continuidades y discontinuidades del tiempo histórico, con el propósito de comprender el presente. A la dificultad de la comprensión amplia y compleja de la temporalidad en el aprendizaje de la Historia se sumó el de la pasividad del rol del estudiante.

Para afrontar esta situación en el diseño de la lección experimental se recurrió a las tecnologías de la Web 2.0, como elemento metodológico transversal de las lecciones de experimentación. En las indagaciones sobre los usos y criterios de los estudiantes acerca de las TIC, se pudo identificar su gusto e interés por la conectividad y acceso a recursos digitales. Desarrollar el pensamiento histórico demanda la participación y empoderamiento de los estudiantes en sus procesos de aprendizaje. Para desarrollar en los estudiantes, y afianzar en los docentes, la cultura del protagonismo de los estudiantes en los procesos de aprendizaje, la tecnología educativa digital fue un componente de innovación fundamental en las lecciones.

La secuencia didáctica se estructuró en función de la metodología del $A B P$. Esta metodología es considera una de las más pertinentes para desarrollar el aprendizaje autónomo y colaborativo (Maldonado, 2009), y al mismo tiempo fomentar el protagonismo del estudiante. Se orientó el diseño de los proyectos para comunicar sus resultados a través de producciones multimedia, como radio, revesita, periódico online; páginas web, producciones audiovisuales, diseño de páginas de Facebook y demás redes sociales de dominio de los estudiantes. En todas las fases de la Lesson Study se tuvo 
como principios pedagógico-didácticos el Flipped classroom, el enactivismo y conectivismo, articulados a la metodología del ABP, secuencia didáctica. En la formación docente en la era digital es imprescindible incorporar el diseño de recursos de autor como material didáctico (Peña, 2017).

En la fase de enseñar y observar la lección los docentes del colectivo prepararon las condiciones del ambiente de aula para minimizar el efecto distorsionador de las filmaciones y observaciones directas en el aula en el comportamiento y el ambiente de estudiantes y docentes. A los estudiantes se les explicó la finalidad de esa Lessón Study y su proceso metodológico. Se desarrolló la primera lección según el diseño. Se socializó con los estudiantes la propuesta de la metodología del ABP.

Los estudiantes trabajaron en grupos y llevaron al aula y otros espacios de aprendizaje, como foros y chats: dudas, dificultades, recursos, hallazgos y resultados de aprendizaje a ser socializados en grupos más amplios y plenarios. De este modo los estudiantes se empoderaron de su rol de protagonistas, principalmente a través del trabajo cooperativo entre pares y más autónomo, con respecto al docente. Para lograr esta autonomía jugó un rol determinante la orientación de los docentes en el diseño de los proyectos realizado por los grupos. En este proceso los estudiantes con la guía de los docentes establecieron: temáticas, objetivos, recursos, cronogramas, resultados y responsabilidades.

Los proyectos se trabajaron en grupos, con aportes individuales específicos; aprendieron haciendo tareas de alta complejidad, como lectura crítica de textos y audiovisuales, participando en foros de discusión y retroalimentación, usando TIC y transformándolas en tecnologías para el aprendizaje y el conocimiento (TAC), construyendo informes de resultados en lenguajes multimedia online e interactivos propios de la Web 2.0. Enseñar y aprender en la era digital implica ir de las TIC a las TAC (Sancho, 2008; Granados J, López R, Avello R, Luna D, Luna E, Luna W, 2014). 
El desarrollo de estos procesos supuso altos niveles de desafío y complejidad, que en la mayoría de los casos fue asumido con gran compromiso. Las plenarias para socializar los resultados de los proyectos mostraron que el diseño de la lección experimental era muy eficaz para lograr el objetivo de aprendizaje, la comprensión del presente histórico. Se observó la primera lección experimental concluyó con buenos resultados, en función del objetivo de aprendizaje, sin embargo, se observó que se debía hacer ajustes al proceso didáctico.

\subsection{Primera lección revisada}

Luego del desarrollo de la lección experimental se hizo el análisis y revisión de ésta, con base en las evidencias recogidas en audiovisuales y fichas de observación. Se hizo algunas mejoras a la primera lección, tales como reforzar el proceso de indagación y socialización de los saberes previos de los estudiantes en torno al tema de aprendizaje y el desarrollo de habilidades de pensamiento relacionadas con la misma. En la lección revisada se reformuló los procesos cognitivos orientados a relacionar los saberes previos de los estudiantes con los nuevos aprendizajes, en función de su significatividad, se dedicó más tiempo para la participación y se orientó a la argumentación de los relatos de los estudiantes sobre sus comprensiones previas.

También se revisó la formación de grupos, como un factor de impacto en la calidad de los procesos de aprendizajes autónomos y cooperativos. Se pudo percibir que algunos grupos eran más eficientes y más comprometidos con el trabajo grupal que otros, debido a que los grupos formados por los mismos estudiantes, en algunos casos se distraían en conversaciones de amigos y en otros casos no había complementariedad en las habilidades y competencias requeridas para la tarea grupal. Por ello, se reconstituyeron grupos en función de sus capacidades para aportar cooperativamente al 
grupo. También se orientó para que en las producciones multimedia se use más imágenes, recursos producidos por los mismos estudiantes.

Se desarrolló la lección revisada, se siguió el mismo procedimiento de observación y recogida de evidencias. Los cambios hechos fueron muy eficaces para mejorar el nivel de logro de objetivos de aprendizaje. Se indagó de forma más amplia sobre conocimientos previos de los estudiantes, a través de la ejemplificación, a partir de vivencias personales. Los estudiantes mostraron más dominio y protagonismo en el proceso del ABP. Usaron recursos digitales multimedia mucho más potentes en el ámbito comunicativo, tales como el uso de lenguajes y entornos más interactivos y novedosos, que fueron más allá de lo orientado y recomendado por los profesores en el proceso didáctico.

Los resultados de esta lección fueron mejores que en la lección anterior. Las presentaciones de los estudiantes daban cuenta de la comprensión de cómo los relatos de la historia están trazados en líneas continuas y discontinuas, con la participación de diversos actores históricos. Sin embargo, la comprensión de la determinación del presente y la identidad de los individuos y los pueblos, a partir de esta lectura de la temporalidad en la historia, aún presentaba algunas dificultades, porque esta comprensión implicaba nociones de secuencialidad y causalidad.

\subsection{Segunda lección revisada}

Se revisó nuevamente la primera lección revisada y se insertó en la planificación didáctica actividades orientadas a desarrollar las habilidades de pensamiento orientadas a comprender la secuencialidad y causalidad en los procesos y tiempo históricos. Desde la exploración de los saberes previos, hasta la presentación de los resultados de los proyectos de aprendizaje se desarrollaron de forma intrínsecamente vinculadas las nociones de temporalidad, secuencia, actoría colectiva y causalidad. Con lo que se logró 
una comprensión amplia, compleja y significativa del presente histórico como elemento identitario de los individuos y los pueblos.

En todo el proceso de la Lesson Study se lograron ambientes de aprendizaje, presenciales y virtuales, en los que predominó el trabajo cooperativo entre estudiantes y docentes. Las interrelaciones personales de horizontalidad fueron una constante en todas las observaciones. A pesar de la presencia de las cámaras y observadores hubo siempre un ambiente de mucha naturalidad, como una característica muy marcada del trabajo cooperativo y planificado por procesos y resultados.

Las producciones comunicativas con las que los estudiantes presentaron los resultados de sus proyectos, con un gran despliegue de recursos multimedia, evidenciaron su comprensión de categorías de continuidad y discontinuidad en el tiempo histórico, en temas tales como el legado histórico de las grandes civilizaciones de Oriente, Occidente y el Mundo Andino y las tres guerras del siglo XX.

Se puso especial énfasis en las civilizaciones cañari e inca, por su importancia en la configuración de la identidad ecuatoriana y latinoamericana. Una de los principales resultados a los que se arribó a través de la Lesson Study, es que los estudiantes valoraron como muy importante el haber comprendido que son herederos de grandes civilizaciones, que hay legados que deben ser preservados, y en algunos casos rescatados, y otros que ya son parte de historia, como algo superado. Otro resultado a destacar es haber fomentado el trabajo colaborativo del colectivo de docentes, con el fin de innovar la propia práctica docente.

\section{Conclusiones}

La experiencia de la Lesson Study fue evaluada por estudiantes, docentes como una propuesta altamente innovadora para desarrollar el pensamiento histórico. El impacto de las tecnologías de la Web 2.0 en los 
aprendizajes fue uno de los elementos mejor evaluados por la capacidad de generar aprendizajes cooperativos, enactivos y en conectividad.

El lema de la UNAE, "Todos los días enseñan algo, todos los días aprendes algo" se volvió muy pragmático en el desarrollo de esta Lesson study. Estudiantes y docentes aprendieron a aprender, enseñando y aprendiendo con sus pares y con los docentes, asumiendo su responsabilidad de principal interesado en el qué, cómo, con quién, con qué y en dónde aprende más y mejor. Con esta metodología todos los participantes vivieron una importante experiencia de reflexiones metacognitivas.

La metodología de la Flipped classroom, en esta experiencia demostró ser eficaz para invertir los roles (Pérez, 2012), del estudiante como sólo aprendiente y del docente como sólo enseñante. Ante el desafió de desarrollar complejos procesos de pensamiento los estudiantes aprendieron y enseñaron entre pares y con sus docentes. Desarrollar producciones comunicativas como resultados de proyectos de aprendizaje en lenguajes multimedia motivó a que los docentes aprendieran junto con los estudiantes y con sus pares. Se vivió una verdadera comunidad de aprendizaje.

\section{Referencias}

Asamblea Constituyente (2008). Constitución Política del Ecuador. Montecristi, Ecuador: Registro Oficial 449, 20 de octubre.

Comisión Gestora de la Universidad Nacional de Educación (2015a,b,c). Modelo Pedagógico. Azogues, Ecuador: s.e.

Granados J, López R, Avello R, Luna D, Luna E, Luna W. (2014). Las tecnologías de la información y las comunicaciones, las del aprendizaje y del conocimiento y las tecnologías para el empoderamiento y la participación como Instrumentos de apoyo al docente de la universidad del siglo XXI. Cuba: Medisur, 12(1), págs. 289-294. [Revista en Internet]. Recuperado de: 
http://medisur.sld.cu/index.php/medisur/article/view/2751

Maldonado, M. (2009). Aprendizaje Basado en Proyectos Colaborativos.

Una experiencia en educación superior. Venezuela: Laurus, 28, págs. 158-180.

Ministerio de Educación del Ecuador (2010). Actualización y Fortalecimiento

Curricular de la Educación General Básica. Quito, Ecuador: s.e.

Ministerio de Educación de Ecuador (2012). Lineamientos curriculares para

el bachillerato general unificado. Quito, Ecuador: Mineduc.

Peña, J. (2017). Creación de una Unidad de Apoyo Docente que Integran

la Tecnología Digital para el Diseño de Medios Didácticos. Revista Scientific, 2(5), 66-85. Recuperado de:

https://doi.org/10.29394/scientific.issn.2542-2987.2017.2.5.4.66-85

Pérez, A. (2012). Educarse en la era digital. Madrid, España: Morata.

Sancho, J. (2008). De TIC a TAC, el difícil tránsito de una vocal. Investigación en la escuela 2008, págs. 19-30.

Sanz, S. \& Pérez, M. (2009). La metodología de la Flipped classroom. Valencia, España: s.e.

Sistema Nacional de Nivelación y Admisión (2014). Microcurrículo por áreas de conocimiento. Quito, Ecuador: s.e.

Soto, E. y Pérez, A. (2014a,b,c,d). Las Lesson Study ¿qué son? Cuadernos de pedagogía. España: Universidad de Málaga, págs. 1-9.

Vallejo, R. (2008). En Manual II de Historia del Ecuador. Quito, Ecuador: Corporación Editora Nacional. 


\section{Gladys Isabel Portilla Faicán}

e-mail: gladys.portilla@unae.edu.ec

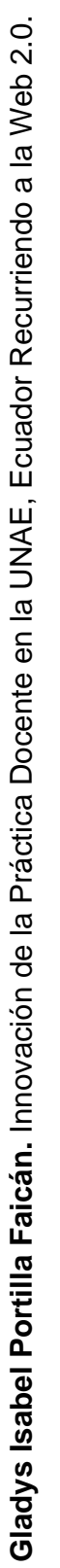

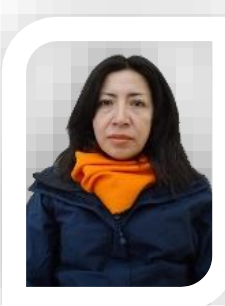

Nacida en Cuenca, Ecuador. Formación de tercer nivel y

cuarto nivel en Ciencias de la Educación y Ciencias

Sociales. Profesora de la Universidad Nacional de

Educación (UNAE-Ecuador). Docente-Investigadora en las áreas de Ciencias sociales, Didáctica de la Ciencias sociales, Formación docente, Innovación educativa y

Tecnología educativa emergente. 


\section{Amauris Laurencio Leyva}

e-mail: amalaur@cepes.uh.cu

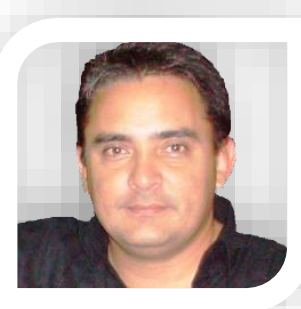

Nacido en La Habana, Cuba. PhD. Presidente de la Comisión de Grados científicos para las Ciencias Sociales de la Universidad de La Habana. Coordinador del Programa de Doctorado en Ciencias de la Educación. Vicedirector del CEPESUH. Cátedra UNESCO en Gestión y Docencia Universitaria. 